\title{
Different aspects of cognitive function in adult patients with moyamoya disease and its clinical subtypes
}

\author{
Zhiyong Shi (D , ${ }^{1}$ Yu-Jie Wen, ${ }^{2,3}$ Zheng Huang, ${ }^{4}$ Le-Bao Yu, ${ }^{1}$ Dong Zhang ${ }^{1}$
}

To cite: Shi Z, Wen Y-J, Huang Z, et al. Different aspects of cognitive function in adult patients with moyamoya disease and its clinical subtypes. Stroke \& Vascular Neurology 2020;5: e000309. doi:10.1136/ svn-2019-000309

- Additional material is published online only. To view, please visit the journal online (http://dx.doi.org/10.1136/svn2019-000309).

Received 29 November 2019 Revised 25 February 2020 Accepted 27 February 2020 Published Online First 21 March 2020

Check for updates

(C) Author(s) (or their employer(s)) 2020. Re-use permitted under CC BY-NC. No commercial re-use. See rights and permissions. Published by BMJ.

${ }^{1}$ Department of Neurosurgery, Beijing Tiantan Hospital, Capital Medical University, Beijing,

China

${ }^{2}$ The National Clinical Research Center for Mental Disorders \& Beijing Key Laboratory of Mental Disorders \& Beijing Institute for Brain Disorders Center of Schizophrenia, Beijing Anding Hospital, Capital Medical University, Beijing, 100088,

China

${ }^{3}$ Advanced Innovation Center for Human Brain Protection, Capital Medical University, Beijing, 100069, China

${ }^{4}$ Department of Neurosurgery, Xiangya Hospital, Xiangya Hospital Central South University, Changsha, China

Correspondence to Dr Dong Zhang; zhangdong0660@aliyun.com

\section{ABSTRACT}

Objective Although a few reports suggested that cognitive function impairment could be found in adult patients with moyamoya disease (MMD), there were still many aspects that are unclear. The aim of our study was to assess the cognitive function of adult patients with MMD and its clinical subtypes.

Methods 49 patients with MMD and 23 healthy controls were asked to take cognitive function tests. Cognitive function tests included $I Q$, prospective memory (PM), immediate memory (IM), verbal fluency (VF), visual breadth, attention, retrospective memory (RM), Stroop test, Wisconsin Card Sorting Test, Trail-Making Test Part A (TMT-A) and Continuous Performance Test (CPT). Independent t-analysis, one-way analysis of variance and Pearson correlation were used to seek for differences between subgroups and the correlation between cognitive variables.

Results Compared with healthy controls, adult patients with MMD had a comprehensive cognitive impairment, including IQ, PM, VF, attention, RM, Stroop, CPT and TMT-A, with more serious impairment in PM and attention. PM and RM were separated, indicating that they were independent of each other. Pattern of attention was significantly different from healthy controls. Female patients were better than male patients, where significant differences in PM, IM, Stroop and WCST could be found. The haemorrhagic patients exhibited poorer in the dimension of PM and RM than the ischaemic. The headache subtype exhibited poorer than healthy controls. PM, RM, attention and executive function were moderately correlated with each other. Conclusions Adult patients with MMD had a wide range of cognitive impairment with more serious impairment in memory and attention. Differences in cognitive function existed between the different subtypes of adult MMD.

\section{INTRODUCTION}

Moyamoya disease (MMD), discovered in 1957, was classically defined as progressive stenosis or occlusion of the bilateral internal carotid artery (ICA) and/or anterior cerebral artery (ACA) and/or middle cerebral artery (MCA) and/or posterior cerebral artery (PCA, about $30 \%$ ), accompanied by formation of smoky vessels at the base of the brain. At present, the aetiology of MMD is unknown. ${ }^{12}$ MMD occurred much more frequently among women than men, with a sex ratio (women to men) reported approximately as $1.8 .^{3}$
Whether there is a difference in cognitve function between male and female remains unkonwn. Unlike children, adult patients with MMD had variable clinical symptoms, including ischaemic stroke, intracranial haemorrhage, headache and transient ischaemic attack (TIA). However, cognitive dysfunction of MMD had been less well studied in adults than in children. ${ }^{4}$ A few reports suggested that cognitive function impairment, including IQ, processing speed, attention, executive function, visual perception, and reconstruction and language function executive, could be found in adult patients with MMD. ${ }^{5-11}$ However, few literature reported the comparison of memory function, such as prospective memory (PM) and retrospective memory (RM) among subgroups of adult patients with MMD. PM was the ability of remembering to execute delayed intentions in the future, whereas RM referred to the recalling of past events and words. ${ }^{12} 13$ Moreover, in our clinical work, a large number of adult patients with MMD complained of occipital headache without ischaemic stroke or intracranial haemorrhage. It had been reported that the incidence of headache in patients with MMD was about $6 \%{ }^{14}$ It was worthwhile to pay attention to these patients with MMD with headache, whose cognitve function and clinical changes were still unclear.

Japan was currentlyconductinga multicentre Cognitive Function Survey on Moyamoya Disease (Cognitive Dysfunction Survey of the Japanese Patients with Moyamoya Disease). ${ }^{15}$ We also wanted to conduct a multicentre cognitive function research on patients with MMD in China. However, this article was an attempt in a single centre. Overall, the aims of our study were as follows: (1) to continuously enrol adult patients with MMD without any surgical interventions and to assess their cognitive function in a single centre in China; (2) to study the changes in cognitive function in adult patients with MMD after operation or conservative treatment; and (3) to further explore the underlying mechanism 
of cognitve function changes with multimodal imaging data (digital subtraction angiography (DSA), CT perfusion (CTP) and functional MRI (fMRI)). By now, the results shown in this article were used to assess cognitive function of adult patients with MMD. The other two aims and multicentre cooperations would be elucidated in the future.

\section{PARTICIPANTS AND METHODS \\ Participant selection}

This study was a prospective study. Diagnostic criteria ${ }^{16}$ for MMD included (1) progressive stenosis or occlusion of the end of ICA and/or ACA/MCA or PCA, (2) formation of smoke vessels at the base of the brain, (3) bilateral sides and (4) unknown aetiology. The admission criteria of patients with MMD and the healthy control group were (1) age $>18$ years, (2) IQ>70, (3) no limb movement disorder, (4) no colour blindness, (5) without any surgical interventions, and (6) no mental disorder and voluntary participants. Exclusion criteria were (1) age $<18$ years, (2) $\mathrm{IQ}<70$, (3) physical dyskinesia, (4) colour blindness, (5) mental disorders, (6) previous revascularisation-treated patients, (7) pregnant women and patients with metal implants in their bodies, who were unsuitable for MRI examination. Since December 2018, adult patients with MMD were enrolled dynamically. Also, healthy people matched with age, sex, regional distribution and education time were recruited as the healthy control group. By now, 49 patients with MMD and 23 healthy controls were enrolled in our study.

\section{Testing procedures}

The examinations of patients with MMD and healthy controls included cognitive function test and MRI examination. All participants signed an informed consent form voluntarily. Cognitive function tests included Adult Wechsler Intelligence Test, PM test, immediate memory (IM) test, verbal fluency (VF) test, visual breadth (VB) test, attention test, RM test, Stroop test, Wisconsin Card Sorting Test (WCST), Trail-Making Test Part A (TMT-A) and Continuous Performance Test (CPT). The duration of cognitive test was about 2.5 hours. All participants were asked to undergo MRI examination, including the sequence of $\mathrm{T} 1$ brain volume, T2 fluid-attenuated inversion recovery, diffusion-weighted imaging, apparent diffusion coefficient, arterial spin labelling, territorial arterial spin labelling, diffusion tensor imaging and resting-state fMRI fMRI. This procedure lasted for about 45 min. Patients with MMD also received routine preoperative examinations such as DSA and CTP of brain to collect preoperative data and to evaluate the treatment strategy (bypass surgery?/indirect surgery?/combined surgery?/conservative treatment). At the follow-up of 6 months after operation, patients with MMD were asked to receive MRI, CTP examination and the cognitive function assessment to evaluate therapeutic effect. The result shown in this paper was the assessment of cognitive function of adult patients with MMD.

Participant selection and testing procedure were simplified, as shown in figure 1.

\section{Cognitive function assessment}

\section{Simple Wechsler Adult Intelligence Test}

This test was divided into four parts, including general knowledge subscale, similarity subscale, visual puzzle subscale and block design subscale. ${ }^{17}$ The scores of subscales were converted into standard scores and converted into IQ.

\section{PM test: CAMPROMPT test}

\section{Procedures}

At the beginning of the test, the tester explained to the testee. ${ }^{18}$ In this process, the participant had to take $25 \mathrm{~min}$ to complete several tasks. During this test, the tester would ask the testee to remember some instructions. The alarm was demonstrated to the participant and the timer was set to $20 \mathrm{~min}$. Time would gradually decrease from 20 to -5 min. The 5 min after the timer set to 0 was represented as ' -5 '. First, the participants were shown five items and told their names. After that, the items were hidden in five different places in the testing room. When the tester told the participants the test was over, the participants told the tester what the five things were and where they are hidden. Second, the tester was reminded to take the key with 7 min left in the alarm. Next, when you met a question about Huangshan (or Mount Taishan), gave me this book. The 'start' button was pressed on the alarm. The partcipants began to do puzzles. When the timer displayed $18 \mathrm{~min}$, the information card was given to the participant. The participant was asked to give the tester this information card $5 \mathrm{~min}$ before the test. When the timer displayed $16 \mathrm{~min}$, participant was told to give me this pen after $7 \mathrm{~min}$. When the timer showed $15 \mathrm{~min}$, the participant was asked to remind the tester to take a call 5 min after the timer was 0 .

\section{Scoring system}

When the participants met Huangshan or Taishan keywords or the timer showed $9,7,5,0$ and $-5 \mathrm{~min}$, respectively, we should observe the patient's response. If the patient had no response, after Huangshan or Taishan question or the timer showed $8,6,4.5,-0.5,-5.5 \mathrm{~min}$, we should tip the participant and observe his or her response.

Situation 1: participant performed a task actively. Grade A: task correct. Grade B: task error, tipped, task correct. Grade C: task error, tipped, still error.

Situation 2: participant forgot the task. Grade D: tipped, task correct. Grade E: tipped, task error, tipped again, task correct. Grade F: tipped, task error, tipped again, task error again.

Situation 3: participant had no response. Grade G: tipped, no response, tipped again, task correct. Grade H: tipped, no response, tipped again, no response or task error again. 


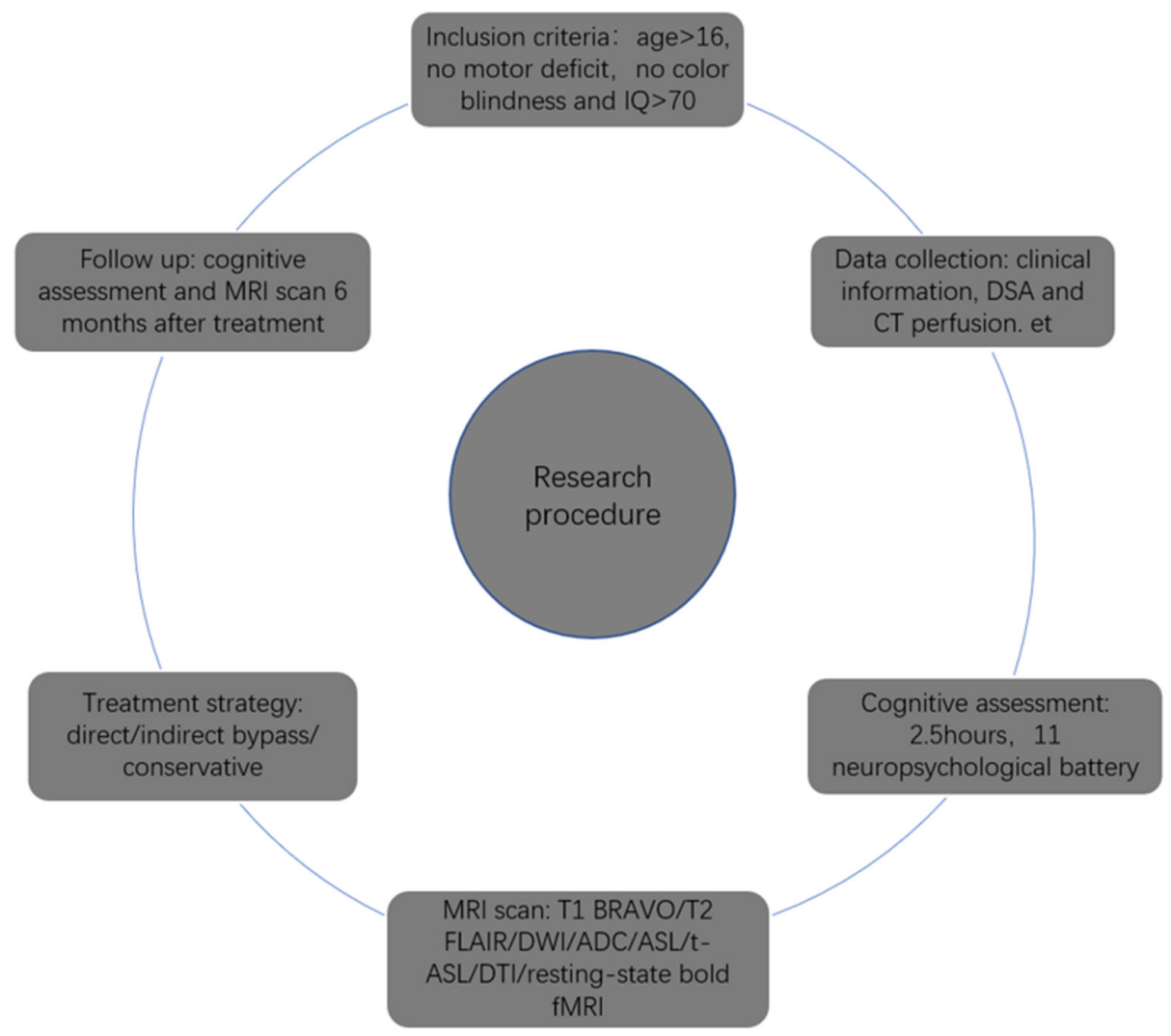

Figure 1 Simplified research procedure in our study. ADC, apparent diffusion coefficient; ASL, arterial spin labelling; BRAVO, brain volume; DSA, digital subtraction angiography; DTI, diffusion tensor imaging; DWI, diffusion-weighted imaging; FLAIR, fluid-attenuated inversion recovery; fMRI, functional MRI; t-ASL, territorial arterial spin labelling.

Score conversion system: $\mathrm{A}=6, \mathrm{~B}=4, \mathrm{C}=2, \mathrm{D}=4, \mathrm{E}=2, \mathrm{~F}=1$, $\mathrm{G}=1, \mathrm{H}=0$.

Time-based tasks included pen change, keys and taking a call (three items). Event-based tasks included books, information card and five-item names and their hidden places (three items). The total score of time-based or event-based tasks was the sum of their scores, respectively. The total score of PM was the sum of time-based prospective memory (TBPM) and event-based prospective memory (EBPM).

\section{Repeatable Battery for the Assessment of Neuropsychological Status (RBANS) Test}

This test had five parts, including IM, VB, VF, attention and RM. IM included list learning and story memory. ${ }^{19}$ VB included picture copy and line orientation. VF included picture naming and semantic fluency. Attention included digital breadth and coding tests. RM included sum of list/story/figure and list recognition. All the test scores of RBANS were original scores, which needed to be converted to scale scores. Then, the total score was the sum of five scale scores. Finally, the scale scores are converted to the total scale of index scores and the percentage of the population.

\section{Stroop test}

The Stroop test included four test items: single word, colour, word interference and colour interference. ${ }^{20}$ The recording of test results included the number of errors and testing time.

\section{Trail-Making Test Part $A$}

(1) Connecting line test: connected dot symbols with straight lines in order (1-24) and the test time (s) is recorded. (2) Puzzle test: in this puzzle, a line is drawn from the beginning to the end and the time (s) is recorded. (3) Finding difference test: 10 differences are determined between the two pictures and the correct number and time (s) are recorded. (4) Judged correct and error number test: within $7 \mathrm{~min}$, each description was followed by pairs of *\# or \#* symbols. Judging whether The order of symbols and descriptions were judged whether they were the same and the number of correct, error and the total was recorded. ${ }^{21}$

\section{Wisconsin Card Sorting Test}

This test was a representative tool for frontal executive function, including conceptual formation, abstract reasoning, flexibility, problem-solving, maintaining goal and sustained attention. ${ }^{22}{ }^{23}$ The computerised version was administered according to standardised instructions. A number of stimulus cards were presented on screen. The keywords on the cards differed with respect to colour, quantity and shape. The participants were instructed to match the cards to sort in a particular way but they were not told the rules for sorting, instead they were told 
whether a particular match is right or wrong on a screen. The test generated a number of psychometric scores, including trials administered, total correct responses, per cent correct responses, total errors, per cent errors (PEs), perseverative responses, per cent perseverative responses, perseverative errors (PEs), per cent perseverative errors, non-perseverative errors, per cent non-perseverative errors, conceptual-level responses, per cent conceptual level responses, categories completed (CCs), trials to compete first category, failure to maintain set, learning to learn and time use (ms). PE and CG were common indicators used in clinical practice.

\section{Continuous Performance Test}

Participants are told to concentrate. The system would give instructions within $720 \mathrm{~s}$. The participants are instructed to follow the instructions and to click the corresponding number with the mouse. The correct number, error number, omission number and reaction time (ms) are recorded. ${ }^{24}$

\section{Attention research}

The paradigm was a well-established central cueing paradigm by Posner et al. ${ }^{25}$ The task was a reaction time button-press test where a target would appear following a central cue, which was indicated by an arrow. The target could either appear on the cued side, constituting a valid condition, or on the opposite side, resulting in an invalid condition. Lastly, the cue can be a bidirectional arrow yielding no indication of the target side, thus being a control condition. By now, 23 adult patients with MMD and 21 healthy controls matched with gender, age, regional distribution and education time were enrolled and compared.

\section{Statistical analysis}

First, we used $\chi^{2}$ test for gender and independent sample t-test or univariate analysis of variance (ANOVA) for age and education time to ensure that there were no significant differences between patients with MMD and healthy controls, patients with haemorrhagic MMD and ischaemic MMD, as well as three ischaemic subgroups. Independent sample t-test for cognitive function comparison between patients with MMD and healthy controls and patients with haemorrhage or ischaemia were used. A univariate ANOVA was used to compare the cognitive function between three different ischaemic presentations (TIA, infarction and headache). Pearson correlation analysis was used to determine the correlation between cognitive variables. The level of statistical significance was set at 0.05. Statistical analysis was performed with SPSS V.19.0 software.

\section{RESULTS}

A total of 49 adult patients with MMD (24 males and 25 females) and 23 healthy controls (9 males and 14 females) were recruited into our study. The ages of patients with MMD and healthy controls were $38.45 \pm 8.99$ and
Table 1 Characteristics of participants and clinical information on patients

\begin{tabular}{lllcl}
\hline & MMD & HC & $\chi^{2} / \mathbf{t}$ & P value \\
\hline $\begin{array}{l}\text { Gender } \\
\text { Male }\end{array}$ & 24 & 9 & 0.612 & 0.46 \\
$\quad$ Female & 25 & 14 & & \\
\hline Age & $38.45 \pm 8.989$ & $37.57 \pm 9.144$ & -0.387 & 0.700 \\
$\begin{array}{l}\text { Education time } \\
\text { (year) }\end{array}$ & $13.35 \pm 2.884$ & $14.22 \pm 2.088$ & 1.452 & 0.152 \\
\hline
\end{tabular}

Type of MMD

\begin{tabular}{lll}
\multicolumn{1}{c}{ Haemorrhagic } & 12 & - \\
\hline Ischaemic & 37 & - \\
TIA & 11 & \\
Infarction & 21 & \\
Headache & 5 & \\
\hline
\end{tabular}

Data are given as mean \pm SD.

$\mathrm{HC}$, healthy control; MMD, moyamoya disease; TIA, transient ischaemic attack.

$37.57 \pm 9.14$ years old, respectively. The education times of patients with MMD and healthy controls were $13.35 \pm 2.88$ and $14.22 \pm 2.09$ years. No significant difference between gender, age and education time could be found (table 1). Moreover, the numbers of adult patients with MMD with haemorrhage and ischaemia were 12 and 37 cases, respectively. The numbers of patients with MMD with TIA, infarction and headache were 11, 21 and 5 cases. Characteristics of participants and clinical information on patients are shown in table 1.

Differences in test performances between adult patients with MMD and healthy controls are shown in figure 2 and online supplementary material table 1 .

Compared with the healthy controls matched by gender, age and education time, the adult MMD had a wide range of cognitive impairment, including $\mathrm{IQ}(\mathrm{t}=3.161, \mathrm{p}=0.002)$, PM $(\mathrm{t}=4.164, \mathrm{p}=0.000), \mathrm{VF}(\mathrm{t}=3.896, \mathrm{p}=0.000)$, attention $(\mathrm{t}=5.129, \mathrm{p}=0.000)$, RM $(\mathrm{t}=2.820, \mathrm{p}=0.006)$, Stroop $(\mathrm{t}=-3.523, \mathrm{p}=0.001)$, CPT $(\mathrm{t}=-3.100, \mathrm{p}=0.003)$ and TMT-A $(\mathrm{t}=-4.329, \mathrm{p}=0.000)$.

Because of the significant difference in IQ between patients with MMD and healthy controls, variable control (covariance analysis) was applied to IQ. TBPM ( $\mathrm{t}=8.339$, $\mathrm{p}=0.005)$, EBPM ( $\mathrm{t}=4.353, \mathrm{p}=0.041)$, attention $(\mathrm{t}=9.883$, $\mathrm{p}=0.002), \mathrm{VF} \quad(\mathrm{t}=4.431, \mathrm{p}=0.039)$ of adult patients with MMD were significantly lower than those of healthy controls. Though a significant difference in PM could be found between two groups, there was no significant difference in the RM ( $\mathrm{t}=1.121, \mathrm{p}=0.293)$. PM and $\mathrm{RM}$ were separated, indicating that PM and RM were independent of each other, as shown in table 2 and figure 3 .

Differences in test performances between male and female patients with MMD are shown in figure 4 and online supplementary material tables 1 and 3 ).

The cognitive functions of female patients were better compared with male ones, where significant differences in PM (TBPM: $\mathrm{t}=-2.431, \mathrm{p}=0.019$; EBPM: $\mathrm{t}=-2.268, \mathrm{p}=0.028$ ), 


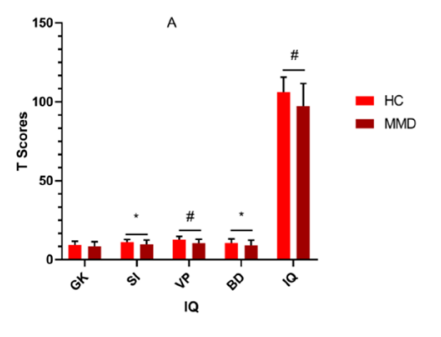

D

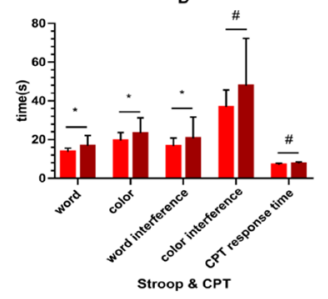

B

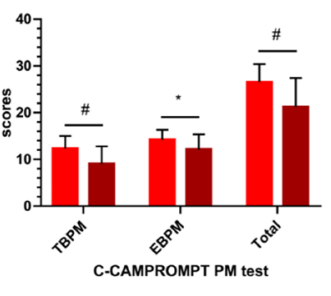

E

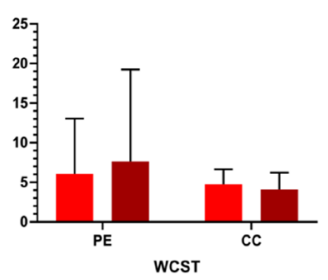

c
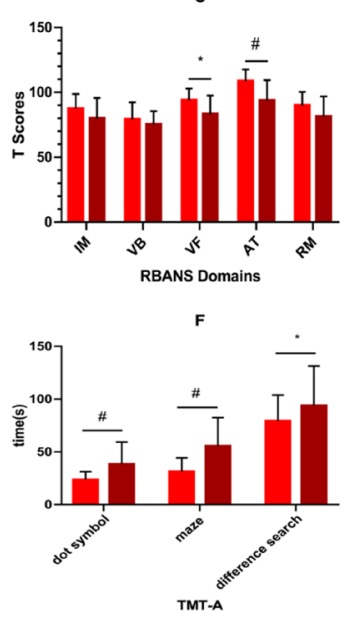

Figure 2 Comparison of cognitive function between MMD and healthy controls. Compared with the healthy controls matched by gender, age and education time, the adult MMD had a wide range of cognitive impairment, including IQ, PM (both TBPM and EBPM), VF, AT, time dimension of Stroop, CPT and TMT-A. Details may be found in online supplementary material table 4. ${ }^{*}, p<0.05 ; \#, p<0.01$. AT, attention; BD, block design; C-CAMPROMPT, Cambridge Prospective Memory Test; CC, category completed; CPT, Continuous Persistence Test; EBPM, event-based prospective memory; GK, general knowledge; HC, healthy control; IM, immediate memory; MMD, moyamoya disease; PE, perseverative error; PM, prospective memory; RBANS, Repeatable Battery for the Assessment of Neuropsychological Status; RM retrospective memory; SI, similarity; TBPM, timebased prospective memory; TMT-A, Trail-Making Test Part A; VB, visual breadth; VF, verbal fluency; VP, visual puzzle; WCST, Wisconsin Card Classification Test.

IM $(\mathrm{t}=-2.902, \mathrm{p}=0.006)$, Stroop $(\mathrm{t}=3.460, \mathrm{p}=0.001)$ and WCST-CC $(\mathrm{t}=-2.645, \mathrm{p}=0.011)$ could be found. However, no similar results could be found between male and female in healthy controls $(\mathrm{p}>0.05)$.

Differences in cognitive function among the different clinical subtypes are shown in figures 5 and 6 and online supplementary material tables 4-6).

Compared with the cognitive function of the ischaemic and haemorrhagic adult patients with MMD, differences in the dimensions of EBPM $(t=2.684, p=0.011)$ and $R M$ $(\mathrm{t}=2.074, \mathrm{p}=0.044)$ could be observed. The other dimensions were not significant $(\mathrm{p}>0.05)$. The cognitive function of adult MMD in the ischaemic group was the headache subtype, which performed better than TIA subtype, which was better than infarction ones. However, no significant difference between three ischaemic subtypes could be found ( $p>0.05$ ). Moreover, the cognitive function of the headache subtype performed worse than that of healthy controls, but there was no significant difference between these two groups $(\mathrm{p}>0.05)$.

The conversion of attention pattern for adult patients with MMD is shown in figure 7 .

The time of attention for adult patients with MMD was significantly higher than healthy controls, including valid, invalid and control patterns $(\mathrm{p}<0.05)$ (figure 6A). However, for adult patients with MMD, the time of valid was higher than the invalid pattern, indicating that the attention pattern had changed, though no significant difference could be found between three subgroups ( $>0.05)$ (figure 6B) .

The association among memory, attention, executive function, VF, gender, age and IQ is shown in table 3.
In the patients group, ТВPM was mainly related to gender $(r=0.334, p<0.05)$, age $(r=-0.363, p<0.05)$, IM $(\mathrm{r}=0.444, \mathrm{p}<0.01), \quad \mathrm{RM} \quad(\mathrm{r}=0.421, \mathrm{p}<0.01)$, attention $(r=0.440, p<0.01)$, total score of RBANS $(r=0.477, p<0.01)$ and WCST-CC $(\mathrm{r}=0.460, \mathrm{p}<0.01)$. EBPM was mainly related to gender $(\mathrm{r}=0.314, \mathrm{p}<0.05)$, IM $(\mathrm{r}=0.382, \mathrm{p}<0.01)$, RM $\quad(\mathrm{r}=0.422, \quad \mathrm{p}<0.01)$, attention $(\mathrm{r}=0.341, \quad \mathrm{p}<0.05)$, total score of RBANS $(\mathrm{r}=0.416, \mathrm{p}<0.01)$ and WCST-CC $(\mathrm{r}=0.428, \mathrm{p}<0.01) \quad($ table $3 \mathrm{~A})$. These results indicated that PM depended on RM, attention and executive function in adult patients with MMD. Attention was mainly related to age $(\mathrm{r}=-0.295, \mathrm{p}<0.05)$, IQ $(\mathrm{r}=0.539, \mathrm{p}<0.01)$, EBPM $(\mathrm{r}=0.341, \mathrm{p}<0.05)$, ТВPM $(\mathrm{r}=0.440, \mathrm{p}<0.01), \mathrm{IM}(\mathrm{r}=0.492$, $\mathrm{p}<0.01), \mathrm{VB}(\mathrm{r}=0.354, \mathrm{p}<0.05), \mathrm{VF}(\mathrm{r}=0.414, \mathrm{p}<0.01), \mathrm{RM}$ $(\mathrm{r}=0.458, \quad \mathrm{p}<0.01)$ and WCST-CC $(\mathrm{r}=0.382, \mathrm{p}<0.01)$ (table 3B). Attention of adult patients with MMD relied on IQ memory and executive function. PM, RM, attention and executive function of adult MMD were correlated with each other, but mainly to a moderate degree.

\section{DISCUSSION}

The highlights of this study were as follows. First, up to now, this study was the most comprehensive assessment of cognitive function of adult patients with MMD in China, including IQ, PM, IM, RM, VF, VB, attention and executive function (CPT, Stroop, WCST and TMT-A). Second, this study found that cognitive function of adult patients with MMD was significantly impaired, with the most serious impairment in PM and attention (pattern changed), which were worthwhile to research further. Third, executive dysfunction in adult patients with MMD 
Table 2 Comparison of cognitive function between MMD and $\mathrm{HCs}$ after IQ controlled (covariance analysis)

\begin{tabular}{lccll}
\hline & HC $(\mathbf{n}=\mathbf{2 3})$ & MMD $(\mathbf{n}=\mathbf{4 9})$ & F & P value \\
\hline PM & & & & \\
TBPM & $12.30 \pm 2.704$ & $9.06 \pm 3.761$ & 8.339 & $.005^{*}$ \\
EBPM & $14.22 \pm 2.110$ & $12.14 \pm 3.208$ & 4.353 & $.041^{*}$ \\
\hline Total & $26.52 \pm 3.860$ & $21.20 \pm 6.232$ & 8.424 & $.005^{*}$ \\
scores & & & & \\
RBANS domains & & & & \\
IM & $87.43 \pm 11.317$ & $79.94 \pm 15.715$ & 0.480 & 0.491 \\
VB & $79.26 \pm 13.084$ & $75.49 \pm 10.017$ & 0.473 & 0.494 \\
VF & $94.04 \pm 8.931$ & $83.27 \pm 14.319$ & 4.431 & $.039^{*}$ \\
AT & $108.70 \pm 8.977$ & $93.76 \pm 15.625$ & 9.883 & $.002^{* *}$ \\
RM & $90.04 \pm 10.227$ & $81.41 \pm 15.381$ & 1.121 & 0.293 \\
SS & $459.48 \pm 34.927$ & $413.86 \pm 53.027$ & 6.023 & $.017^{*}$ \\
TSIS & $88.57 \pm 8.727$ & $77.63 \pm 12.667$ & 5.916 & $.018^{*}$ \\
WCST & & & & \\
\hline PE & $10.87 \pm 24.125$ & $12.10 \pm 24.636$ & 0.022 & 0.881 \\
CC & $4.74 \pm 1.912$ & $4.10 \pm 2.114$ & 0.013 & 0.910 \\
\hline
\end{tabular}

Data are given as mean \pm SD.

iBoldfaced values: If $\mathrm{P}$ value is lower than 0.05 , boldfaced was used to discriminate.

${ }^{*} \mathrm{P}<0.05,{ }^{*} \mathrm{P}<0.01$.

AT, attention; CC, category completed; EBPM, event-based prospective memory; $\mathrm{HC}$, healthy control; IM, immediate memory; MMD, moyamoya disease; PE, perseverative error; PM, prospective memory; RBANS, Repeatable Battery for the Assessment of Neuropsychological Status; RM, retrospective memory; SS, scale score; TBPM, time-based prospective memory; TSIS, total scale of index scores; VB, visual breadth; VF, verbal fluency; WCST, Wisconsin Card Classification Test.

exhibited abnormalities in time dimensions, suggesting that adult patients with MMD might have visual-related attention impairment. Fourth, onset syptoms and gender were associated with memory or executive function, with poorer performances for haemorrhagic or male patients with MMD. Oestrogen might be a protective factor for cognitve funtion of adult patients with MMD. Fifth, headache-type adult patients with MMD caused by brain ischaemia, mostly classified as asymptomatic, performed worse than healthy controls. In the ischaemic subgroups, patients with MMD with headache performed better than patients with TIA, who performed better than patients with infarction. However, no significant difference could be seen between these three subgroups.

In this preliminary study, we found a wide range of cognitive impairment in patients with MMD, which was consistent with previous research conclusions. ${ }^{4} 5$ 26-28 Karzmark et al studied cognitive function in 36 cases of MMD and found that the incidence of cognitive impairment was about $31 \%(11 / 36)$, but most degree of them were mild. ${ }^{6}$ Kronenburg et al found that the incidence of cognitive impairment in adult MMD was about 30\%, and the median IQ was 95 (94-99).$^{29}$ In our study, the average IQ of adult MMD was $97(97.27 \pm 14.36)$, while the average value of healthy controls was $106(106.26 \pm 9.44)$. The IQ of adult MMD group was significantly lower than that of healthy controls, and adult patients with MMD had poorer performance in similarity, visual puzzles and block design. Inaddition, we also found that adult patients with MMD had poor performances in memory, $\mathrm{VF}$, executive function, attention and visual perception, which was consistent with previous studies. ${ }^{6-11}$ After covariance analysis of IQ, PM and attention abnormality of adult patients with MMD could still be found, while VB, $\mathrm{VF}$ and executive function had no significant difference.

For PM impairment, we speculated that PM impairment was more obvious in adult patients with MMD, whereas RM and IM impairment were not obvious. Previous literature had reported that PM impairment was mainly related to prefrontal cortex (PFC) injury ${ }^{30}$ and RM was related to hippocampus or other memory circuits. ${ }^{31}$ The interactions between the hippocampus and the PFC also had a crucial role in episodic memory. ${ }^{32}$ Bilateral lesions of either the PFC or the hippocampus produced a memory deficit in a particular task, while unilateral lesions were typically found to spare the studied aspect of memory, indicating that a unilateral combination of these areas and the pathways between them was sufficient to support memory. ${ }^{33}$ Our study found that adult patients with MMD had PM injury while RM had no injury. We hypothesised
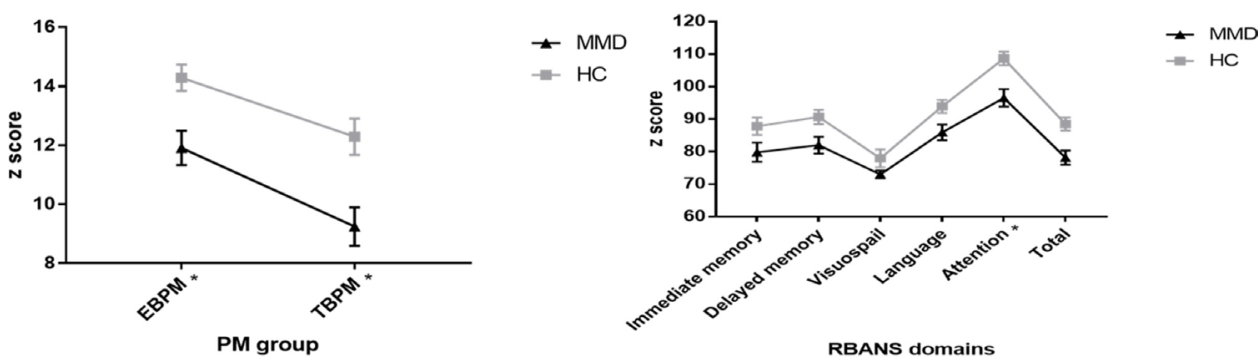

Figure 3 Comparison of cognitive function between MMD and healthy controls matched with gender, age,regional distribution and education level by covariate analysis (IQ adjusted). After variable controlled (IQ adjusted), significant difference between two groups in EBPM, TBPM and attention dimension could still be found. However, RM had no significant difference between the two groups. The separation between PM and RM indicated that PM and RM were independent of each other. EBPM, eventbased prospective memory; HC, healthy control; MMD, moyamoya disease; PM, prospective memory; RBANS, Repeatable Battery for the Assessment of Neuropsychological Status; RM, retrospective memory; TBPM, time-based prospective memory. 
A

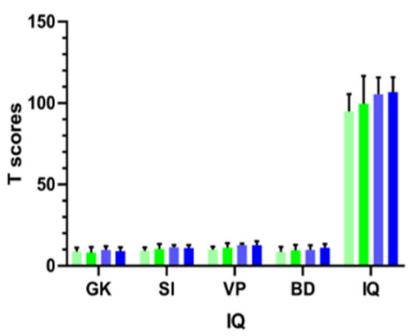

D

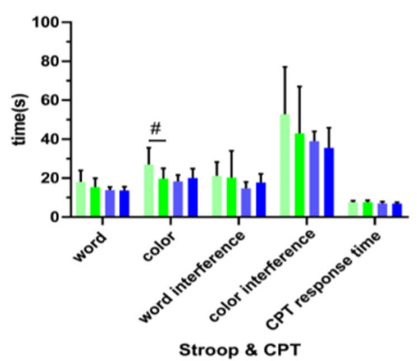

B

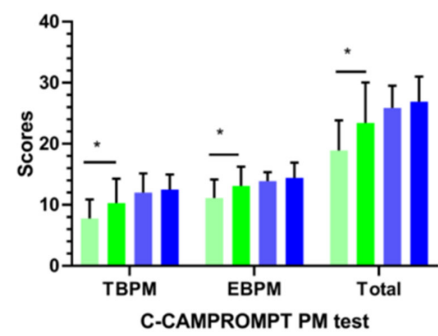

E

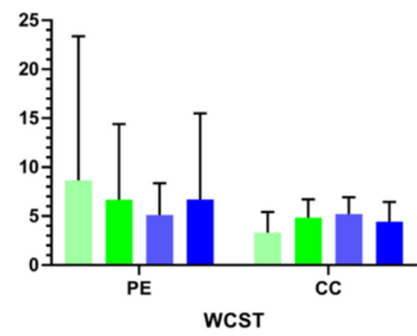

C
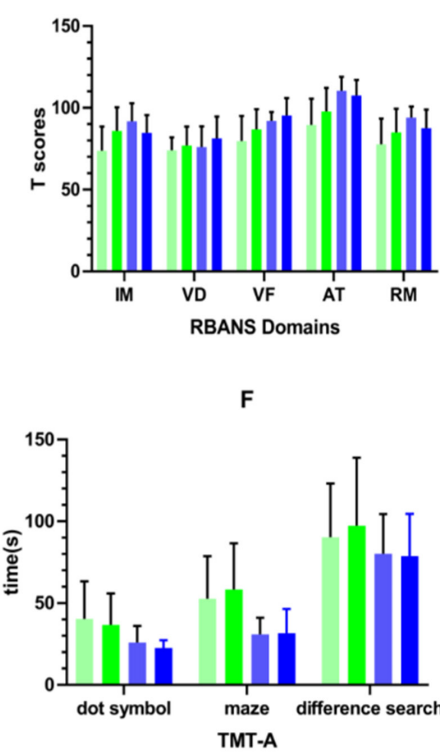

Figure 4 Comparison of cognitive function between male and female adult patients with MMD and HCs. For adult patients with MMD, the cognitive functions of female patients were better compared with male ones, where significant differences in PM (TBPM and EBPM), IM, Stroop and WCST-CC could be found. However, no similar results could be found between male and female in HCs. Details could be found in online supplementary material tables 8 and 9. *, p<0.05; \#, p<0.01. AT, attention; BD, block design; C-CAMPROMPT, Cambridge Prospective Memory Test; CC, category completed; CPT, Continuous PersistenceTest; EBPM, event-based prospective memory; GK, general knowledge; HC, healthy control; IM, immediate memory; MMD, moyamoya disease; PE, perseverative error; PM prospective memory; RM retrospective memory; SI, similarity; TBPM, time-based prospective memory; TMT-A, Trail-Making Test Part A; VB, visual breadth; VF, verbal fluency; VP, visual puzzle; WCST, Wisconsin Card Classification Test.

that cognitive function damage caused by MMD might be mainly in bilateral prefrontal lobe or hippocampus prefrontal circuit, while the hippocampus-limbic system, located in temporal lobe mainly, was perfused by compensatory arteries from posterior circulation, transdural collateral circulation or moyamoya vessels with the progression of MMD. Thus, the cognitive function impairment was occurred, whereas the degree was mild or moderate, indicating the separation of PM and RM. Moreover, this assumption should be supported by resting state fMRI, which would be elucidated in our future research.

However, the working pattern of attention for adult patients with MMD was also significantly different from matched healthy controls, where the invalid pattern was superior than the valid pattern for MMD, whereas the pattern for the healthy was just the opposite. The conversion of attention pattern for adult patients with MMD had no similar reports before. We hypothesised that attention pattern conversion was caused by the insufficient brain perfusion and conversion of cerebral perfusion pattern because of the progressive stenosis of intracranial artery and formation of moyamoya vessels or compensatory artery. As for the exact cerebral region or circuit, it was still a mystery. Whether the attention conversion could be changed by surgical interventions should be elucidated in our future research.
During goal-oriented tasks, attention played a role in visual processing at multiple sites in the central nervous system, from initial visual target selection to high level executive processes. ${ }^{34}$ However, the attention of patients with MMD was significantly impaired in our study. We had divided attention dimension into active attention and passive attention and designed the corresponding tasks to explore further. As for the correlation analysis of PM and attention, we found PM relied RM and attention and executive function, while attention relied on IQ, $\mathrm{PM}$, attention and executive function. We suspected that attention and PM impairment were more serious in adult patients with MMD. By now, there was no relevant literature to report similar research results, which needed to explore further.

Besides, a large number of literature reported that there were significant executive dysfunction in subgroups between adult MMD population. ${ }^{9} 1035-37$ However, in our study, significant differences were only found in the time dimension of TMT-A, Stroop and WCST for adult MMD. After IQ variable controlled, no significant difference in WCST between the two groups could be found. The latest meta-analysis reported that the incidence of executive dysfunction in adult MMD was about $38 \%(19 \%-57 \%){ }^{29}$ Moreover, case reports concerning executive function of patients with MMD found that not all executive function were impaired. ${ }^{528} 38$ Therefore, we suspected that 


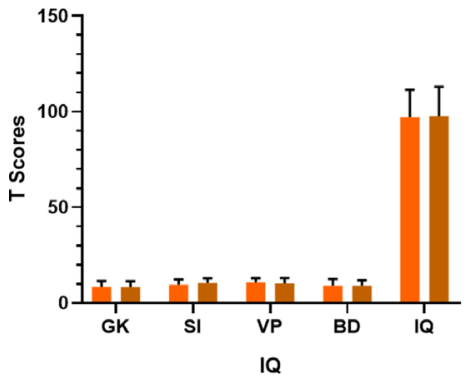

D

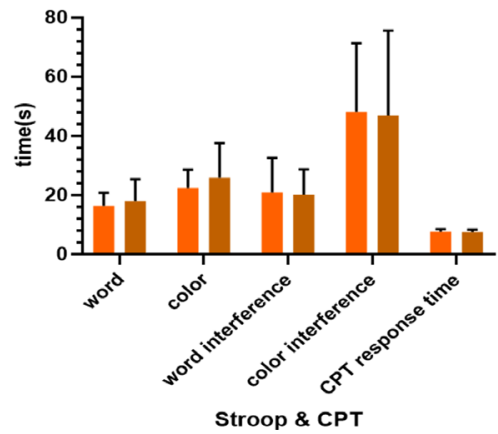

B

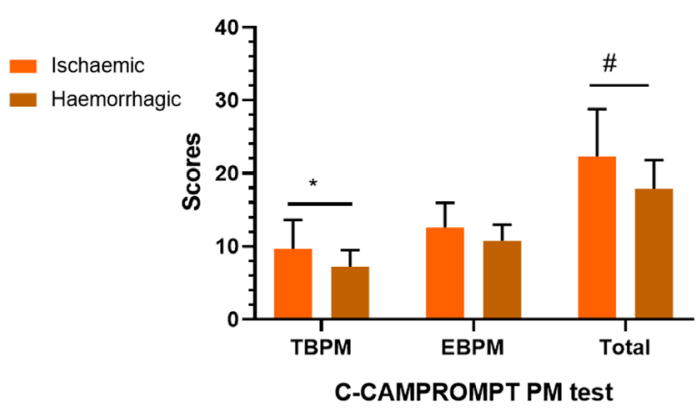

E

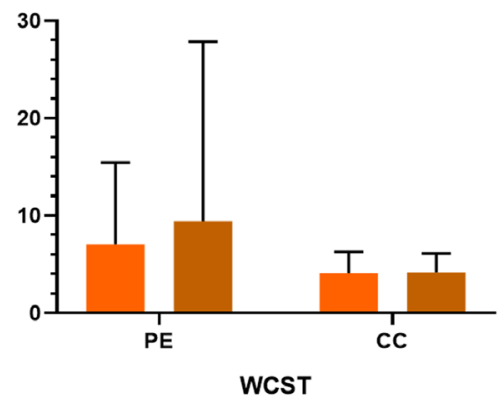

C

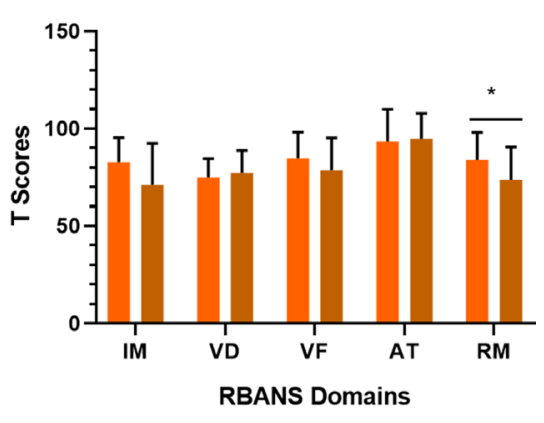

$\mathbf{F}$

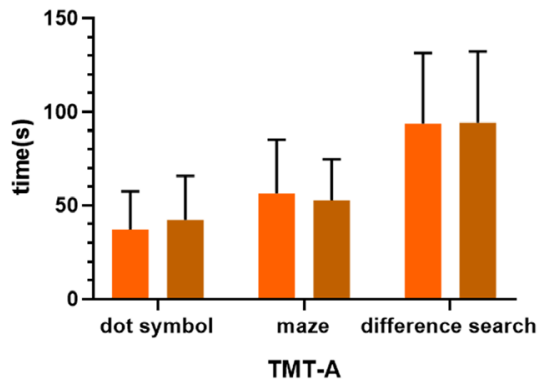

Figure 5 Comparison of cognitive function between ischaemic and haemorrhagic adult patients with moyamoya disease. The ischaemic patients performed better than the haemorrhagic patients, where the differences in the dimensions of EBPM and RM could be observed. The other dimensions were not significant ( $p>0.05)$. Details could be found in online supplementary material table 5. *, $\mathrm{p}<0.05$; \#, $\mathrm{p}<0.01$. AT, attention; BD, block design; BPM, event-based prospective memory; C-CAMPROMPT, Cambridge Prospective Memory Test; CC, category completed; CPT, Continuous PersistenceTest; GK, general knowledge; IM, immediate memory; PE, perseverative error; PM prospective memory; RBANS, Repeatable Battery for the Assessment of Neuropsychological Status; RM, retrospective memory; SI, similarity; TBPM, time-based prospective memory; TMT-A, TrailMaking Test Part A; VB, visual breadth; VF, verbal fluency; VP, visual puzzle; WCST, Wisconsin Card Classification Test.

executive dysfunction in adult patients with MMD exhibited abnormalities in time dimensions of Stroop, CPT and TMT-A, suggesting that adult patients with MMD might have visual-related attention circuit impairment which should also be supported by fMRI data in our future study.

As for the comparison between ischaemic and haemorrhagic patients with MMD, previous literature reported that the haemorrhagic exhibited worse executive inhibition compared with the ischaemic, whereas the ischaemic had a worse working memory and poorer sustained attention. ${ }^{8}$ In our study, we found that the overall cognitive function of the haemorrhagic was lower than that of the ischaemic, with significant impairment in memory. Moreover, TBPM and RM were significantly worse in haemorrhagic group, while IQ, VF, VB attention, CPT, Stroop, WCST and TMT-A had no significant difference. We hypothesised that haemorrhage related to the fragility or chronic compression of the neovessels led to much more severe impairment than ischaemia. Acute process, such as cerebral haemorrhage, had more serious impact on the cognitve funtion of brain than chronic process, including TIA infarction or headache. Therefore, we suspected that memory impairment of adult patients with MMD was associated with the first onset symptoms, and the haemorrhagic performed worse than the ischaemic.
Furthermore, our study also found that female patients had better cognitive function than male ones, including PM, IM, Stroop and WCST. However, there was no significant difference between male and female in healthy controls. Thus, female patients were superior to male patients in memory and executive function, and no similar results had reported before. This difference in cognitive function between gender might be associated with cerebral perfusion or hormone. Moreover, Hara et al recognised that oestrogen facilitated higher cognitive functions by exerting effects on brain regions such as the PFC and hippocampus. ${ }^{39}$ Oestrogen-induced spinogenesis and synaptogenesis in these two brain regions and also initiated a complex set of signal transduction pathways via oestrogen receptors in the rodent, monkey, and human brain. ${ }^{39} 40$ Therefore, we hypothesised that oestrogen might be a protective factor for cognitive function of adult patients with MMD.

In addition, for comparison between subgroup analysis of ischaemic patients with MMD, we recognised that the headache subtype performed better than that of TIA, which performed better than infarction type, which was partially consistent with reports of Fang et al. ${ }^{8}$ We suspected that these findings might be related to the degree of cerebral ischaemia in patients with headache type, while the degree of cerebral ischaemia in patients 
A

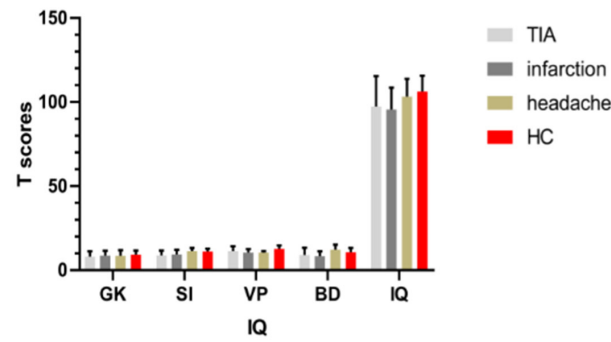

D

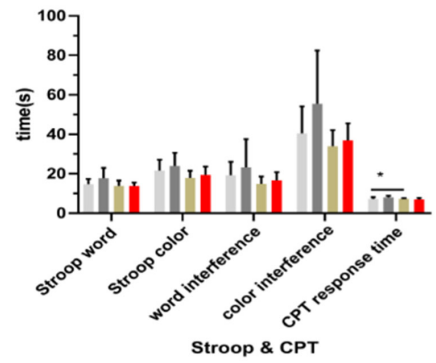

B

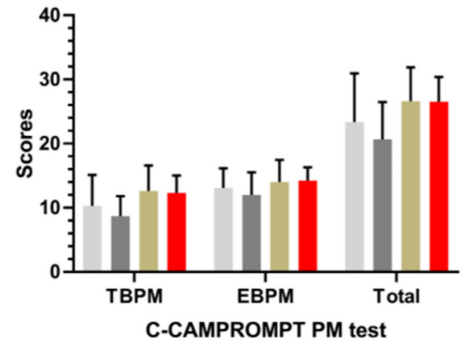

E

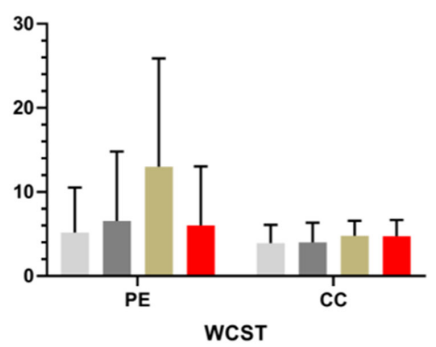

C

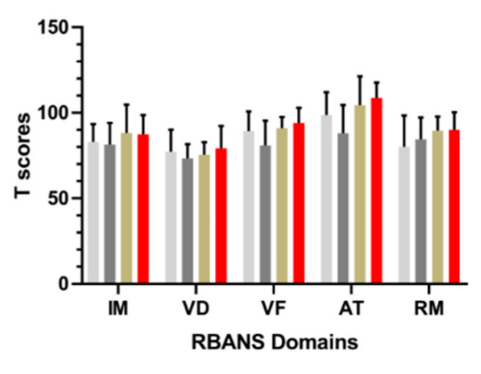

$\mathbf{F}$

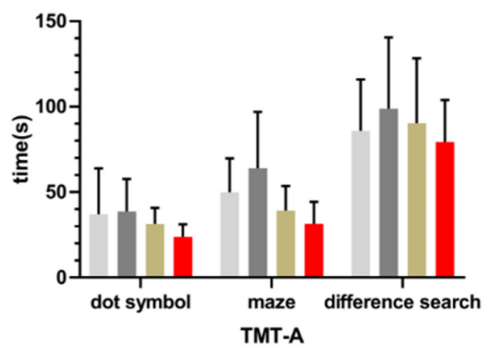

Figure 6 Comparison of cognitive function between ischaemic subtypes for adult patients with moyamoya disease. For ischaemic patients, the headache subtype performed better than the TIA subtype, which was better than infarction ones. However, no significant difference between three ischaemic subtypes could be found ( $>>0.05)$. Moreover, the cognitive function of the headache subtype performed worse than that of $\mathrm{HCs}$, but there was no significant difference between these two groups ( $p>0.05$ ). Details could be found in online supplementary material tables 6 and $7 .{ }^{*}, p<0.05 ; \#, p<0.01$. AT, attention; BD, block design; C-CAMPROMPT, Cambridge Prospective Memory Test; CC, category completed; CPT, Continuous Persistence Test; EBPM, event-based prospective memory; GK, general knowledge; HC, healthy control; IM, immediate memory; PE, perseverative error; PM, prospective memory; RM retrospective memory; SI, similarity; TBPM, time-based prospective memory; TIA, transient ischaemic attack; TMTA, Trail-Making Test Part A; VB, visual breadth; VF, verbal fluency; VP, visual puzzle; WCST, Wisconsin Card Classification Test.

with headache type was the lightest. It had been reported that the incidence of headache in moyamoya patients was about $6 \%(57 / 962),{ }^{14}$ and the incidence in our study was $10 \%(5 / 49)$. Previous literature reported that adult patients with MMD had headache as their potential complaints and were suspected to have suffered from TIA in their childhood, which spontaneously resolved during their adolescence by the reduced demand of cerebral blood flow in their adolescence and adulthood. Such
A

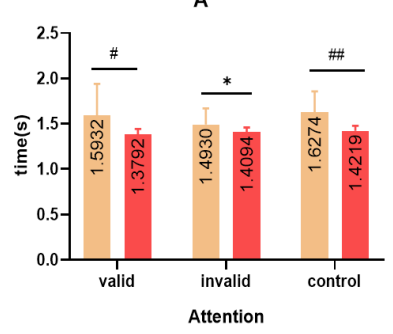

B

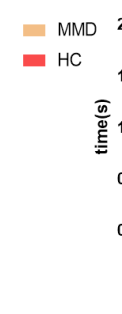

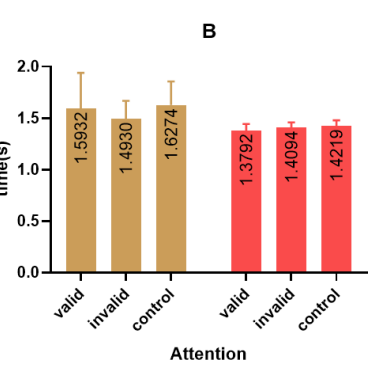

Attention
Figure 7 Analysis of attention pattern for adult patients with MMD and HCs. The time of attention for adult patients with MMD was significantly higher than healthy controls, including valid, invalid and control patterns (A). For adult patients with MMD, the time of the valid pattern was higher than that of the invalid pattern, indicating that attention pattern had changed, though no significant difference could be found between the three subgroups (B). ${ }^{*}, \mathrm{p}<0.05$; \#, $\mathrm{p}<0.01$. HC, healthy control; MMD, moyamoya disease. patients sometimes complain of headache without ischaemic symptoms even after the disappearance of TIA. ${ }^{41} 42$ Thus, we should pay more attention to ischaemic patients with headache complaints in the clinic.

The shortage of this study was in the following aspects: (1) the overall sample size, including patients with MMD and healthy controls, was too small for subgroup classification, which might bring about statistical bias; next, we needed to increase the sample size to validate and make our results more compelling and informative, especially for the numbers of the healthy controls; (2) the preliminary results shown here lacked imaging statistics, which needed to be supplemented by DSA, perfusion CT and structural or fMRI data; (3) when gender was associated with cognitive function, the statistical results might not be accurate because of not considering the impact of Suzuki classification and cerebral infarction; multivariate analysis and variables controlled should be considered in our further study with the increase in numbers of participant enrolment; (4) the value of SD in WCST-PE was very large, which could contribute to statistical bias; the reasons included that three patients with brain infarction could not understand the WCST test intentions and resulted in extreme values; moreover, the sample size was small and needed to be further expanded; (5) the results of this study were the preliminary results of a single centre; further results needed to be further improved after the 
Table 3 Correlation between gender, age, education time, IQ, PM, RBANS domains and WCST for adult patients with MMD

(A) Showed correlation between PM and gender, age, education time, IM, VB, VF, AT, RM, SS, PE and CC

\begin{tabular}{|c|c|c|c|c|c|c|c|c|c|c|c|c|}
\hline & Gender & Age & $\begin{array}{l}\text { Education } \\
\text { time }\end{array}$ & IQ & IM & VB & VF & AT & RM & SS & PE & CC \\
\hline TBPM & $0.334^{*}$ & $-0.363^{*}$ & 0.104 & $0.312^{*}$ & $0.444^{\star *}$ & 0.127 & 0.258 & $0.440^{* *}$ & $0.421^{\star *}$ & $0.477^{* *}$ & -0.116 & $0.460^{* *}$ \\
\hline
\end{tabular}

SS, Scale score.

\begin{tabular}{lllllllllllll}
\hline \multicolumn{10}{l}{ (B) Exhibited correlation between AT and gender, age, IQ, PM, RM, VB, VF, RM, SS, PE and CC } \\
\hline AT & Gender & Age & IQ & TBPM & EBPM & RM & VB & VF & RM & SS & PE & CC \\
\hline
\end{tabular}

${ }^{*} \mathrm{P}<0.05,{ }^{* *} \mathrm{P}<0.01$.

AT, attention; CC, category completed; EBPM, event-based prospective memory; IM, immediate memory; MMD, moyamoya disease; PE, perseverative error; PM, prospective memory; RBANS, Repeatable Battery for the Assessment of Neuropsychological Status; RM, retrospective memory; TBPM, time-based prospective memory; VB, visual breadth; VF, verbal fluency; WCST, Wisconsin Card Classification Test.

multicentre data aggregation; and (6) Healthy people were established as comparison group for the cognitive damage caused by ischaemic stroke or haemorrhage for adult patients with MMD. However, the cognitive unique profile for MMD was still unkown. The comparison group of other adult stroke patients with non-MMD aetiology should be considered in our future study.

\section{CONCLUSIONS}

Adult patients with MMD and its different subtypes had a wide range of cognitive impairment, with more serious impairment in memory and attention compared with healthy controls. There was a separation between PM and $\mathrm{RM}$, indicating that PM and RM were independent of each other, while PM, RM, attention and executive function of patients with MMD were moderately correlated with each other. The memory function of adult MMD might be related to the onset symptoms and gender, with poor performance for the haemorrhagic and male patients. Abnormalities in time dimensions of Stroop, CPT and TMT-A suggest that adult patients with MMD might have visual-related attention circuit impairment.

Contributors ZS: first author; design and conceptualisation the study, analysis of the data and drafting of the manuscript for intellectual content. Y-JW: cofirst author; data collection and analysis, drafting and revision of manuscript. DZ: corresponding author; drafting and revision for intellectual content (appendix 2). L-BY,

coinvestigator, director of coordinating centre; led and coordinated communication among participants. ZH, coinvestigator and site investigator; reviewed all protocols and statistical deviations.

Funding The authors have not declared a specific grant for this research from any funding agency in the public, commercial or not-for-profit sectors.

Competing interests None declared.

Patient consent for publication Not required.

Ethics approval This study was approved by the ethics committee of Tiantan Hospital.

Provenance and peer review Not commissioned; externally peer reviewed.

Data availability statement All data relevant to the study are included in the article or uploaded as supplementary information. All data in my research would be uploaded in my original article and would be available for the editors. Anyone who needed this supplementary materials could contact with Dr Zhiyong Shi ( szy1195156829@aliyun.com).

Open access This is an open access article distributed in accordance with the Creative Commons Attribution Non Commercial (CC BY-NC 4.0) license, which permits others to distribute, remix, adapt, build upon this work non-commercially, and license their derivative works on different terms, provided the original work is properly cited, appropriate credit is given, any changes made indicated, and the use is non-commercial. See: http://creativecommons.org/licenses/by-nc/4.0/.

ORCID iD

Zhiyong Shi http://orcid.org/0000-0003-3392-0041

\section{REFERENCES}

1 Scott RM, Smith ER. Moyamoya disease and moyamoya syndrome. N Engl J Med 2009;360:1226-37.

2 Araki Y, Takagi Y, Ueda K, et al. Cognitive function of patients with adult moyamoya disease. J Stroke Cerebrovasc Dis 2014;23:1789-94.

3 Kuriyama S, Kusaka Y, Fujimura M, et al. Prevalence and clinicoepidemiological features of moyamoya disease in Japan: findings from a nationwide epidemiological survey. Stroke 2008;39:42-7.

4 Matsushima Y, Aoyagi M, Masaoka $\mathrm{H}$, et al. Mental outcome following encephaloduroarteriosynangiosis in children with moyamoya disease with the onset earlier than 5 years of age. Child's Nerv Syst 1990;6:440-3.

5 Jefferson AL, Glosser G, Detre JA, et al. Neuropsychological and perfusion MR imaging correlates of revascularization in a case of moyamoya syndrome. AJNR Am J Neuroradiol 2006;27:98-100.

6 Karzmark P, Zeifert PD, Tan S, et al. Effect of moyamoya disease on neuropsychological functioning in adults. Neurosurgery 2008;62:1048-52.

7 Hoare AM, Keogh AJ, disease CM. Cerebrovascular moyamoya disease. Br Med J 1974;1:430-2.

8 Fang L, Huang J, Zhang Q, et al. Different aspects of dysexecutive syndrome in patients with moyamoya disease and its clinical subtypes. J Neurosurg 2016;125:299-307.

9 Calviere L, Ssi Yan Kai G, Catalaa I, et al. Executive dysfunction in adults with moyamoya disease is associated with increased diffusion in frontal white matter. J Neurol Neurosurg Psychiatry 2012;83:591-3.

10 Festa JR, Schwarz LR, Pliskin N, et al. Neurocognitive dysfunction in adult moyamoya disease. J Neurol 2010;257:806-15.

11 Kazumata K, Tha KK, Narita $\mathrm{H}$, et al. Chronic ischemia alters brain microstructural integrity and cognitive performance in adult moyamoya disease. Stroke 2015;46:354-60.

12 de Mendonça A, Felgueiras H, Verdelho A, et al. Memory complaints in amnestic mild cognitive impairment: more prospective or retrospective? Int J Geriatr Psychiatry 2018;33:1011-8. 
13 Einstein GO, McDaniel MA. Normal aging and prospective memory. $J$ Exp Psychol Learn Mem Cogn 1990;16:717-26.

14 Suzuki J, Takaku A. Cerebrovascular "moyamoya" disease. Disease showing abnormal net-like vessels in base of brain. Arch Neurol 1969;20:288-99.

15 Takagi Y, Miyamoto S, Group CO-JS. Cognitive dysfunction survey of the Japanese patients with moyamoya disease (COSMO-JAPAN study): study protocol. Neurol Med Chir 2015;55:199-203.

16 Fukui M. Guidelines for the diagnosis and treatment of spontaneous occlusion of the circle of Willis ('moyamoya' disease). Research Committee on spontaneous occlusion of the circle of Willis (moyamoya disease) of the Ministry of health and welfare, Japan. Clin Neurol Neurosurg 1997;99 Suppl 2:S238-40.

17 Chinese Map Press. Wechsler adult intelligence Scale-Chinese version manual. Changsha, China: Chinese Map Press, 1992.

18 Li X, Wang K, Jia S, et al. The prospective memory of patients with idiopathic REM sleep behavior disorder. Sleep Med 2018;47:19-24.

19 Loughan AR, Braun SE, Lanoye A. Repeatable battery for the assessment of neuropsychological status (RBANS): preliminary utility in adult neuro-oncology. Neurooncol Pract 2019;6:289-96.

20 Lee TM, Chan CC. Stroop interference in Chinese and English. J Clin Exp Neuropsychol 2000;22:465-71.

21 Tombaugh TN. Trail making test a and B: normative data stratified by age and education. Arch Clin Neuropsychol 2004;19:203-14.

22 Gardizi E, King JP, McNeely HE, et al. Comparability of the WCST and WCST-64 in the assessment of first-episode psychosis. Psychol Assess 2019;31:271-6.

23 McMillan SG, Rees CS, Pestell C. An investigation of executive functioning, attention and working memory in compulsive hoarding. Behav Cogn Psychother 2013;41:610-25.

24 AJDA-Ohoo C. Associations between neuroticism and executive function outcomes: response inhibition and sustained attention on a continuous performance test.

25 Posner MI, Snyder CR, Davidson BJ. Attention and the detection of signals. J Exp Psychol 1980;109:160-74.

26 Sato H, Sato N, Tamaki N, et al. Chronic low-perfusion state in children with moyamoya disease following revascularization. Childs Nerv Syst 1990;6:166-71.

27 Lehman RA, Chelune GJ, Heaton RK. Level and variability of performance on neuropsychological tests. J Clin Psychol 1979;35:358-63.
28 Lubman DI, Pantelis C, Desmond P, et al. Moyamoya disease in a patient with schizophrenia. J Int Neuropsychol Soc 2003;9:806-10.

29 Kronenburg A, van den Berg E, van Schooneveld MM, et al. Cognitive functions in children and adults with moyamoya vasculopathy: a systematic review and meta-analysis. J Stroke 2018;20:332-41.

30 Szczepanski SM, Knight RT. Insights into human behavior from lesions to the prefrontal cortex. Neuron 2014;83:1002-18.

31 Scoville WB, Milner B. Loss of recent memory after bilateral hippocampal lesions. J Neurol Neurosurg Psychiatry 1957;20:11-21.

32 Place R, Farovik A, Brockmann M, et al. Bidirectional prefrontalhippocampal interactions support context-guided memory. Nat Neurosci 2016;19:992-4.

33 Eichenbaum H. Prefrontal-hippocampal interactions in episodic memory. Nat Rev Neurosci 2017;18:547-58.

34 Baluch F, Itti L. Mechanisms of top-down attention. Trends Neurosci 2011;34:210-24.

35 Calviere L. Correlation between cognitive impairment and cerebral hemodynamic disturbances on perfusion magnetic resonance imaging in European adults with moyamoya disease. Clinical article.

36 Nakamizo A, Kikkawa Y, Hiwatashi A, et al. Executive function and diffusion in frontal white matter of adults with moyamoya disease. J Stroke Cerebrovasc Dis 2014;23:457-61.

$37 \mathrm{SH}$ Set al. Assessment of cognitive function in adult patients with hemorrhagic moyamoya disease who received no surgical revascularization.

38 Bornstein RA. Neuropsychological performance in Moya Moya disease: a case study. Int J Neurosci 1985;26:39-46.

39 Hara Y, Waters EM, McEwen BS, et al. Estrogen effects on cognitive and synaptic health over the lifecourse. Physiol Rev 2015;95:785-807.

40 Mitchnick KA, Mendell AL, Wideman CE, et al. Dissociable involvement of estrogen receptors in perirhinal cortex-mediated object-place memory in male rats. Psychoneuroendocrinology 2019;107:98-108.

41 Minelli C, Takayanagui OM, dos Santos AC, et al. Moyamoya disease in Brazil. Acta Neurol Scand 1997:95:125-8.

42 Kuroda S, Hashimoto N, Yoshimoto T, et al. Research Committee on moyamoya disease in J. radiological findings, clinical course, and outcome in asymptomatic moyamoya disease: results of multicenter survey in Japan. Stroke 2007;38:1430-5. 